\title{
Impact of Randomized Trials Comparing Conventional and Endovascular Abdominal Aortic Aneurysm Repair on Clinical Practice
}

\author{
Annette F. Baas, PhD1; Diederick E. Grobbee, MD, $\mathrm{PhD}^{1}$; and \\ Jan D. Blankensteijn, MD, PhD²
}

1Julius Center for Health Sciences and Primary Care, University Medical Center Utrecht, The Netherlands. ${ }^{2}$ Department of Surgery, University Medical Center St. Radboud, The Netherlands.

\begin{abstract}
Purpose: To report a retrospective study into the effects of trials on clinical decisionmaking regarding abdominal aortic aneurysm (AAA) patients suitable for both conventional open (OR) and endovascular aneurysm repair (EVAR).

Methods: A questionnaire was sent to 1400 Dutch surgeons and trainees. Interviewees had to choose between OR and EVAR for AAA patients with and without comorbidity. Specifically, their preferences before and after the publication of 2 randomized trials (EVAR-1 and DREAM) were polled.

Results: Of the $524(37 \%)$ questionnaires returned, $223(43 \%)$ respondents treated AAA patients. Before publication of the trials, $160(72 \%)$ preferred OR for the patient without comorbidity and $169(76 \%)$ preferred EVAR for the patient with comorbidity. In total, 72 $(32 \%)$ respondents changed their preference after the trials were published; however, there was no overall major shift. Focusing on the different cases revealed that the OR preference was significantly enhanced for the patient without comorbidity $(p<0.01)$, while the EVAR preference was significantly enhanced for the patient with comorbidity $(p<0.05)$.

Conclusion: The randomized trials have not induced major overall changes in surgical decision-making for AAA patients suitable for both EVAR and OR.
\end{abstract}

J Endovasc Ther 2007;14:536-540

Key words: abdominal aortic aneurysm, endovascular aneurysm repair, open repair, randomized controlled trials, questionnaire

Rupture rates rise as abdominal aortic aneurysms (AAA) expand, so the threshold for surgical intervention is usually $55 \mathrm{~mm}$ in diameter. ${ }^{1}$ Currently, there are 2 methods to treat AAA: conventional open repair (OR) or endovascular aneurysm repair (EVAR). Prospective European and British registries have shown lower perioperative mortality after EVAR compared to OR. ${ }^{2,3}$ However, there is an increased risk of complications and re- interventions, mainly caused by incomplete exclusion of the AAA and structural failure of the stent-graft. ${ }^{4}$

The DREAM (Dutch Randomized Endovascular Aneurysm Management) trial was initiated in 1999 to compare the results of OR and EVAR. $^{5}$ In this study, 351 AAA patients anatomically suitable for both procedures were randomized to either OR (178 patients) or EVAR (173 patients). At the same time,

The authors have no commercial, proprietary, or financial interest in any products or companies described in this article.

Address for correspondence and reprints: Annette Baas, UMC Utrecht, Julius Center, PO Box 85500, 3584 CX Utrecht, The Netherlands. Fax: 31-30-250-5480; E-mail: a.f.baas@umcutrecht.nl 
TABLE 1

Responses to the Questionnaire Sent to Dutch Surgeons and Trainees

\begin{tabular}{lcccc}
\hline & Residents $(\mathrm{n}=400)$ & General Surgeons $(\mathrm{n}=650)$ & Vascular Surgeons $(\mathrm{n}=350)$ & Total $(\mathrm{n}=1400)$ \\
\hline Respondents & $131(33 \%)$ & $244(38 \%)$ & $149(43 \%)$ & $524(37 \%)$ \\
Treat AAA patients & $48(37 \%)$ & $26(11 \%)$ & $149(100 \%)$ & $223(43 \%)$ \\
$\begin{array}{l}\text { Vascular surgery is their } \\
\text { primary practice }\end{array}$ & $4(3 \%)$ & $0(0 \%)$ & $149(100 \%)$ & $153(29 \%)$ \\
\hline
\end{tabular}

AAA: abdominal aortic aneurysm.

a British randomized trial was initiated with inclusion criteria similar to the DREAM trial; the EVAR-1 study enrolled 1082 patients. ${ }^{6}$

The short-term results of the DREAM and EVAR-1 trials were almost identical. ${ }^{7,8}$ DREAM reported a postoperative mortality risk in EVAR patients of $1.2 \%$ against $4.6 \%$ in OR patients. The midterm results were also similar. $^{9,10}$ The initial survival advantage of EVAR disappeared after the first postoperative year. Two years after treatment, the survival rates were equal at $\sim 90 \%$.

The impact of the DREAM and EVAR-1 trials on the policy of AAA treatment depends on their assessment within the surgical community. To gain insight into how the results of the trials have affected the opinion of best surgical option for an $A A A$, we devised a questionnaire to survey all surgeons in The Netherlands.

\section{METHODS}

In The Netherlands, both general and vascular surgeons conduct vascular surgery. There are $\sim 1000$ surgeons and 400 surgical trainees. About 350 surgeons are certified members of the Dutch Society for Vascular Surgery. In November 2005, a blinded questionnaire was sent to all surgeons and surgical trainees in The Netherlands. In the questionnaire, the role and fields of interest of the respondents were addressed first. Surgeons who were not taking care of AAA patients were asked to return the survey without addressing the case questions. The following dilemma was presented to respondents who did treat patients with AAA: You observe 2 patients in your clinic with an AAA of $65 \mathrm{~mm}$, both anatomically and clinically perfectly suitable for both conventional and endovascular treatment:
Patient A: 65 years, no comorbidity

Patient $B$ : 77 years, mild comorbidity

Which treatment for either patient did you prefer before the publication of the DREAM and EVAR-1 trials (even if this was a slight and non-scientific preference), and which do you prefer after these trial results appeared?

Differences between groups were compared using the Fisher exact test; $p<0.05$ was considered statistically significant.

\section{RESULTS}

Of the $\sim 1400$ questionnaires sent, 524 (37\%) were returned. Of these, $223(43 \%)$ indicated that they treated AAA patients (Table 1). The answers, stratified by position (Table 2), show no major differences in outcome among the different groups. Before publication of the DREAM and EVAR-1 trials, 160 (72\%) respondents would have preferred OR and $63(28 \%)$ respondents would have preferred EVAR for the patient without comorbidity. After publication of the results, 164 (74\%) respondents preferred OR and 59 (26\%) EVAR. For the patient with comorbidity, 54 $(24 \%)$ respondents preferred OR and 169 (76\%) preferred EVAR before the trials were published. After publication, 58 (26\%) preferred OR and 165 (74\%) preferred EVAR.

In all, $72(32 \%)$ respondents changed their opinion on AAA treatment of patient $A$ or patient $B$ after the publication of the DREAM and EVAR-1 trials (Fig. 1). The pre-trial preference of $O R$ for patient $A(72 \%)$ was enhanced (Fig. 2), as significantly more vascular surgeons changed their opinion from EVAR to OR $(17 / 46,37 \%)$ than from OR to EVAR $(11 / 103,11 \% ; p<0.01)$. The pre-trial preference of EVAR for patient B (76\%) was 
TABLE 2

Results of the Questionnaire to Dutch Surgeons and Trainees Who Treat AAA Patients

\begin{tabular}{|c|c|c|c|c|c|c|c|c|}
\hline & \multicolumn{2}{|c|}{ Residents ( $n=48$ ) } & \multicolumn{2}{|c|}{ Surgeons $(n=26)$} & \multicolumn{2}{|c|}{ Vascular Surgeons $(n=149)$} & \multicolumn{2}{|c|}{ Total $(n=223)$} \\
\hline & OR & EVAR & OR & EVAR & OR & EVAR & OR & EVAR \\
\hline \multicolumn{9}{|l|}{ Patient A* } \\
\hline Before trials $\uparrow$ & $39(81 \%)$ & $9(19 \%)$ & $18(69 \%)$ & $8(31 \%)$ & $103(69 \%)$ & $46(31 \%)$ & $160(72 \%)$ & $63(28 \%)$ \\
\hline After trials & $37(77 \%)$ & $11(23 \%)$ & $18(69 \%)$ & $8(31 \%)$ & $109(73 \%)$ & $40(27 \%)$ & $164(74 \%)$ & $59(26 \%)$ \\
\hline \multicolumn{9}{|l|}{ Patient B } \\
\hline Before trials & $11(23 \%)$ & $37(77 \%)$ & 8 (31\%) & $18(69 \%)$ & $35(24 \%)$ & $114(76 \%)$ & $54(24 \%)$ & 169 (76\%) \\
\hline After trials & $12(25 \%)$ & $36(75 \%)$ & 8 (31\%) & $18(69 \%)$ & $38(25 \%)$ & 111 (75\%) & $58(26 \%)$ & 165 (74\%) \\
\hline
\end{tabular}

OR: open repair, EVAR: endovascular aneurysm repair.

* Patient A: 65 years old and no comorbidity; Patient B: 77 years old with mild comorbidity.

$\dagger$ EVAR-1 and DREAM.

enhanced significantly by residents and vascular surgeons $(p<0.05)$.

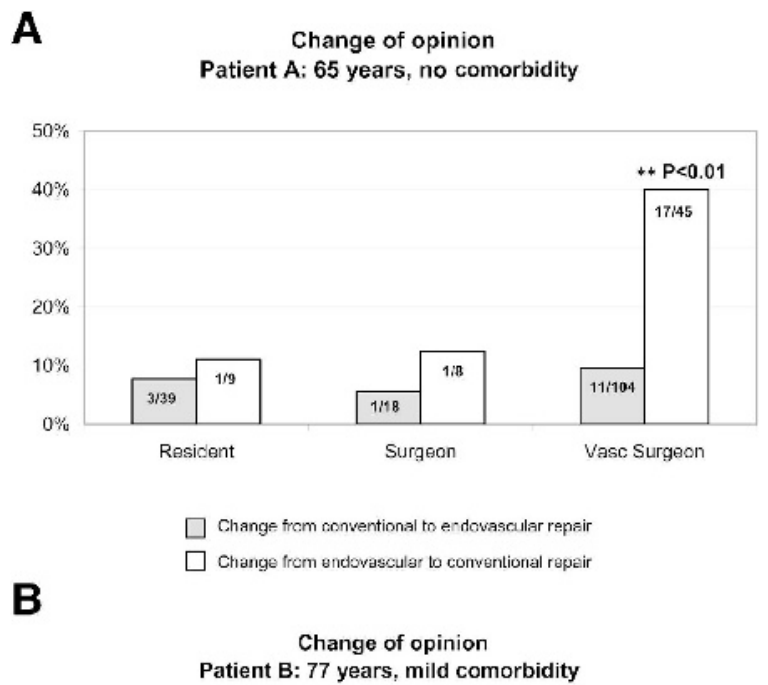

The differences in interpretation of the randomized trials comparing conventional and endovascular AAA repair among the trialists and the vascular surgery community have been remarkable. On one hand, better perioperative results after EVAR compared to OR combined with a similar midterm mortality risk can be used to claim superiority of EVAR over OR. On the other hand, one may argue that EVAR has no midterm advantages, is more expensive, has a higher reintervention rate, and is therefore inferior to OR. Clearly, the trials have not established an absolute preference for EVAR or OR, which is emphasized by the results of our questionnaire.

\section{Change of opinion patient $\mathrm{A}$ and/or $\mathrm{B}$}

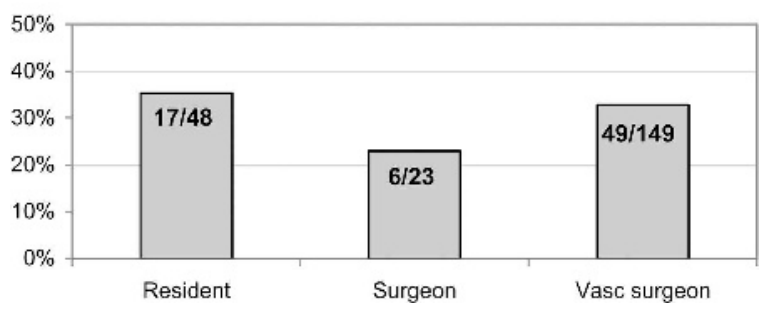

Figure $1 \diamond$ Percentages of respondents who changed their opinion on clinical policy of AAA treatment after the publication of the randomized trials for patient $A$ (without comorbidity) and/or patient $B$ (with comorbidity).

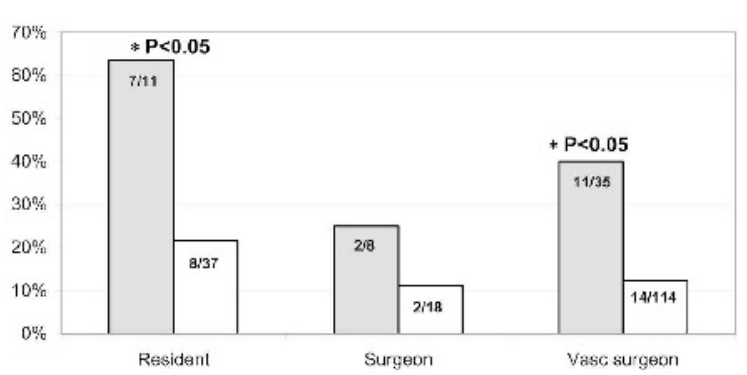

Figure $2 \triangleleft$ Percentages of respondents who changed their opinion on clinical policy of AAA treatment after the publication of the randomized trials for (A) patient $A$ (without comorbidity) and (B) patient $\mathrm{B}$ (with comorbidity). The respondents who preferred OR before the trials and EVAR after the trials are represented by the gray bars; the respondents who preferred EVAR before the trials and OR after the trials are represented by the white bars. 
Although about one third of the respondents changed their opinion after publication of the randomized trials, this did not lead to a significant overall change of AAA treatment policy. Before and after the trials appeared in print, $\sim 75 \%$ of the respondents preferred OR for the patient without comorbidity and EVAR for the patient with comorbidity.

However, when focusing on the individual cases, we noticed interesting shifts. Before the trials, the major proportion of surgeons preferred OR for the patient without comorbidity (patient A). Among the minority of surgeons who favored EVAR for this patient, $31 \%$ changed their opinion after the trials in favor of OR, while only $9 \%$ of the OR supporters switched to EVAR. This is quite surprising as the trials do not provide evidence that OR may be more beneficial for this patient group. Actually, a recent subgroup analysis of the DREAM trial suggested that the opposite might be true; younger ( $\leq 70$ years) EVAR-treated patients had lower 2-year mortality rates that younger OR-treated patients. ${ }^{11}$ Likewise, the EVAR-1 trialists have suggested that the fitter the patient, the more the benefit that can be gained from EVAR over OR. A plausible explanation for the outcome of the questionnaire is that it is believed that younger, more fit patients who are likely to survive the invasive OR procedure, live longer and thereby have a higher chance of undergoing reinterventions that would make EVAR less beneficial. Clearly, the randomized trials have not provided evidence for this assumption.

For patient B, the elderly patient with comorbidity, the pre-trial preference was $76 \%$ for EVAR. Of the surgeons who voted against EVAR, 37\% changed their opinion after the trials in favor of EVAR, while only $14 \%$ of the EVAR supporters switched their preference to OR, which suggests that the trials have reduced even further the concern for EVAR treatment in this patient group despite the absence of trial evidence to support this notion. This may be due to the fact that the trials did show that there are no major short-term EVAR-related complications, and that most prior EVAR opponents feared these complications in the older patient group.

\section{Limitations}

Due to the retrospective nature of the study, it can be argued that it might have been hard for the respondents to remember their pretrials opinion. However, we feel that the methods used have assessed the perceived individual change of opinion, which is a notable measure in itself. Whether this individual perceived change of mind is an accurate representation of the actual change of point of view from pre- to post-trials will remain unknown.

Another limitation to our study design is the low response rate $(37 \%)$, although this is not uncommon for surveys such as this one. We carefully assume that the surgeons who are willing to read and return the survey are the surgeons who recognize the importance of clinical trials and the need for exploring their impact. Almost half of the vascular surgeons responded, and we expect that these surgeons have experience in endovascular surgery. Therefore, the outcome of the survey may not be completely generalizable to the global vascular community, but it will likely reflect the opinion of leading vascular surgeons.

\section{Conclusion}

Based on this questionnaire, we believe that the randomized trials have not induced major overall changes in surgical decisionmaking for AAA patients suitable for both EVAR and OR.

\section{REFERENCES}

1. The UK Small Aneurysm Trial Participants. Mortality results for randomized controlled trial of early elective surgery or ultrasonographic surveillance for small abdominal aortic aneurysms. Lancet. 1998;352:1649-1655.

2. Vallabhaneni SR, Harris PL. Lessons learnt from the EUROSTAR registry on endovascular repair of abdominal aortic aneurysm repair. Eur J Radiol. 2001;39:34-41.

3. Thomas SM, Gaines PA, Beard JD. Short-term (30-day) outcome of endovascular treatment of abdominal aortic aneurism: results from the prospective Registry of Endovascular Treatment of Abdominal Aortic Aneurism (RETA). Eur J Vasc Endovasc Surg. 2001;21:57-64. 
4. van Marrewijk C, Buth J, Harris PL, et al. Significance of endoleaks after endovascular repair of abdominal aortic aneurysm: the EUROSTAR experience. J Vasc Surg. 2002;35: 461-473.

5. Prinssen M, Buskens E, Blankensteijn JD. The Dutch Randomized Endovascular Aneurysm Management (DREAM) trial. Background, design and methods. J Cardiovasc Surg (Torino). 2002;43:379-384.

6. Brown LC, Epstein D, Manca A, et al. The UK endovascular repair (EVAR) trials: design, methodology and progress. Eur J Vasc Endovasc Surg. 2004;27:372-381.

7. Prinssen M, Verhoeven EL, Buth J, et al. A randomized trial comparing conventional and endovascular repair of abdominal aortic aneurysms. N Engl J Med. 2004;351:1607-1618.
8. Greenhalgh RM, Brown LC, Kwong GP, et al. Comparison of endovascular aneurysm repair with open repair in patients with abdominal aortic aneurysm (EVAR trial 1), 30-day operative mortality results: randomised controlled trial. Lancet. 2004;364:843-848.

9. Blankensteijn JD, de Jong SE, Prinssen M, et al. Two-year outcomes after conventional or endovascular repair of abdominal aortic aneurysms. N Engl J Med. 2005;352:2398-2405.

10. EVAR trial participants. Endovascular aneurysm repair versus open repair in patients with abdominal aortic aneurysm (EVAR trial 1): randomised controlled trial. Lancet. 2005;365:2179-2186.

11. Baas $A F$, de Jong $S E$, Prinssen $M$, et al. The benefit of EVAR: role of age. In: Branchereau A, Jacobs M, eds. Vascular Procedures in the Elderly. Oxford: Paris Consultants Ltd.; 2006:95-104. 